\title{
A HYBRID MULTILEVEL/GENETIC APPROACH FOR CIRCUIT PARTITIONING
}

\author{
Charles J. Alpert ${ }^{1}$ \\ Lars W. Hagen ${ }^{2}$ \\ Andrew B. Kahng ${ }^{1}$ \\ ${ }^{1}$ UCLA Computer Science Department, Los Angeles, CA 90095-1596 \\ ${ }^{2}$ Cadence Design Systems, San Jose, CA 94135
}

\begin{abstract}
We present a genetic circuit partitioning algorithm that integrates the Metis graph partitioning package [15] originally designed for sparse matrix computations. Metis is an extremely fast iterative partitioner that uses multilevel clustering. We have adapted Metis to partition circuit netlists, and have applied a genetic technique that uses previous Metis solutions to help construct new Metis solutions. Our hybrid technique produces better results than Metis alone, and also produces bipartitionings that are competitive with previous methods [20] [18] [6] while using less CPU time.
\end{abstract}

\section{INTRODUCTION}

A netlist hypergraph $H(V, E)$ has $n$ modules $V=$ $\left\{v_{1}, v_{2}, \ldots v_{n}\right\}$; a hyperedge (or net) $\in \in E$ is defined to be a subset of $V$ with size greater than one. A bipartitioning $P=\{X, Y\}$ is a pair of disjoint clusters (i.e., subsets of $V$ ) $X$ and $Y$ such that $X \cup Y=V$. The cut of a bipartitioning $P=\{X, Y\}$ is the number of nets which contain modules in both $X$ and $Y$, i.e., $\operatorname{cut}(P)=|\{e \mid e \cap X \neq \emptyset, e \cap Y \neq \emptyset\}|$. Given a balance tolerance $r$, the min-cut bipartitioning problem seeks a solution $P=\{X, Y\}$ that minimizes $\operatorname{cut}(P)$ such that $\frac{n(1-r)}{2} \leq|X|,|Y| \leq \frac{n(1+r)}{2}$.

The standard bipartitioning approach is iterative improvement based on the Kernighan-Lin (KL) [16] algorithm, which was later improved by Fiduccia-Mattheyses (FM) [8]. The FM algorithm begins with some initial solution $\{X, Y\}$ and proceeds in a series of passes. During a pass, modules are successively moved between $X$ and $Y$ until each module has been moved exactly once. Given a current solution $\left\{X^{\prime}, Y^{\prime}\right\}$, the module $v \in X^{\prime}$ (or $Y^{\prime}$ ) with highest gain $\left(=\operatorname{cut}\left(\left\{X^{\prime}-v, Y^{\prime}+v\right\}\right)-\operatorname{cut}(\{X, Y\})\right)$ that has not yet been moved is moved from $X^{\prime}$ to $Y^{\prime}$. After each pass, the best solution $\left\{X^{\prime}, Y^{\prime}\right\}$ observed during the pass becomes the initial solution for a new pass, and the passes terminate when a pass does not improve the initial solution. FM has been widely adopted due to its short runtimes and ease of implementation.

One significant improvement to FM addresses the tiebreaking used to choose among alternate moves that have the same gain. Krishnamurthy [17] proposed a lookahead tie-breaking mechanism, and Sanchis [22] extended this approach to multi-way partitioning. Hagen, Huang, and Kahng [9] have shown that a "last-in-first-out" scheme based on the order that modules are moved in FM is significantly better than random or "first-in-first-out" tiebreaking schemes. More recently, Dutt and Deng [7] independently reached the same conclusion. Finally, Saab [21] has also exploited the order in which modules are moved to produce an improved FM variant.

A second significant improvement to FM integrates clustering into a "two-phase" methodology. A $k$-way clustering of $H(V, E)$ is a set of disjoint clusters $P^{k}=\left\{C_{1}, C_{2}, \ldots C_{k}\right\}$ such that $C_{1} \cup C_{2} \cup \ldots \cup C_{k}=V$ where $k$ is sufficiently large. ${ }^{1}$ We denote the input netlist as $H_{0}\left(V_{0}, E_{0}\right)$. A clustering $P^{k}=\left\{C_{1}, C_{2}, \ldots, C_{k}\right\}$ of $H_{0}$ induces the coarser netlist $H_{1}\left(V_{1}, E_{1}\right)$, where $V_{1}=\left\{C_{1}, C_{2}, \ldots, C_{k}\right\}$ and for every $e \in E_{0}$, the net $e^{\prime}$ is a member of $E_{1}$ where $e^{\prime}=\left\{C_{i} \mid \exists v \in e\right.$ and $\left.v \in C_{i}\right\}$ unless $\left|e^{\prime}\right|=1$ (i.e., each cluster in $e^{\prime}$ contains some module that is in $e$ ). In two-phase FM, a clustering of $H_{0}$ induces the coarser netlist $H_{1}$, and then $\mathrm{FM}$ is run on $H_{1}\left(V_{1}, E_{1}\right)$ to yield the bipartitioning $P_{1}=\left\{X_{1}, Y_{1}\right\}$. This solution then projects to the bipartitioning $P_{0}=\left\{X_{0}, Y_{0}\right\}$ of $H_{0}$, where $v \in X_{0}\left(Y_{0}\right)$ if and only if for some $C_{h} \in V_{1}$, $v \in C_{h}$ and $C_{h} \in X_{1}\left(Y_{1}\right)$. FM is then run a second time on $H_{0}\left(V_{0}, E_{0}\right)$ using $P_{0}$ as the initial solution.

Many clustering algorithms for two-phase FM have appeared in the literature (see [2] for an overview of clustering methods and for a general netlist partitioning survey). Bui et al. [5] find a random maximal matching in the netlist and compact the matched pairs of modules into $\frac{n}{2}$ clusters; the matching can then be repeated to generate clusterings of size $\frac{n}{4}, \frac{n}{8}$, etc. Often, two-phase FM (not including the time needed to cluster) is faster than a single FM run because the first FM run is for a smaller netlist and the second FM run starts with a good initial solution, allowing fast convergence to a local minimum.

The "two-phase" approach can be extended to include more phases; such a multilevel approach is illustrated in Figure 1 (following [15]). In a multilevel algorithm, a clustering of the initial netlist $H_{0}$ induces the coarser netlist $H_{1}$, then a clustering of $H_{1}$ induces $H_{2}$, etc. until the coarsest netlist $H_{m}$ is constructed ( $m=4$ in the Figure). A partitioning solution $P_{m}=\left\{X_{m}, Y_{m}\right\}$ is found for $H_{m}$ (e.g., via FM) and this solution is projected to $P_{m-1}=\left\{X_{m-1}, Y_{m-1}\right\}$.

\footnotetext{
${ }^{1} \mathrm{~A}$ partitioning and a clustering are identical by definition, but the term partitioning is generally used when $k$ is small (e.g., $k \leq 10$ ), and the term clustering is generally used when $k$ is large (e.g., $k=\Theta(n)$ with constant average cluster size). Although a bipartitioning can also be written as $P^{2}=\left\{C_{1}, C_{2}\right\}$, we use the notation $P=\{X, Y\}$ to better distinguish between partitioning and clustering.
} 
$P_{m-1}$ is then refined, e.g., by using it as an initial solution for FM. In the Figure, each projected solution is indicated by a dotted line and each refined solution is given by a solid dividing line. This uncoarsening process continues until a partitioning of the original netlist $H_{0}$ is derived.

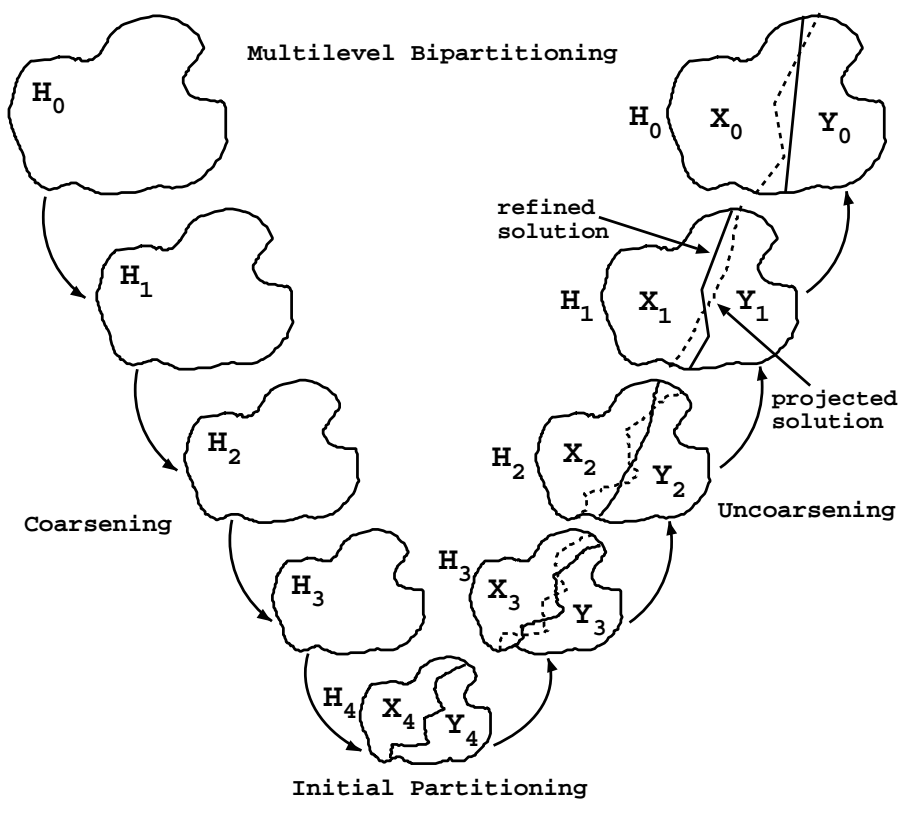

Figure 1. The multilevel bipartitioning paradigm.

Multilevel clustering methods have been virtually unexplored in the physical design literature; the work of Hauck and Borriello [10] is the notable exception. [10] performed a detailed study of multilevel partitioning for FPGAs and found that simple connectivity-based clustering combined with a KL and FM multilevel approach produced excellent solutions. However, multilevel partitioning has been wellstudied in the scientific computing community, e.g., Hendrickson and Leland [11] [12] and Karypis and Kumar [13] [14] [15] have respectively developed the Chaco and Metis partitioning packages.

The Metis package of [15] has produced very good partitioning results for finite-element graphs and is extremely efficient, requiring only 2.8 seconds of CPU time on a Sun Sparc 5 to bipartition a graph with more than 15,000 vertices and 91,000 edges. Our initial hypothesis, which our work has verified, was that Metis adapted for circuit netlists is both better and faster than FM. Metis runtimes are so low that we can easily afford to run it over 100 times to generate a partitioning. However, instead of simply calling Metis 100 times, we propose to integrate Metis into a genetic algorithm; our experiments show that this approach produces better average and minimum cuts than Metis alone. Overall, our approach generates bipartitioning solutions that are competitive with the recent approaches of [20] [18] [6] while requiring much less $\mathrm{CPU}$ time.

The rest of our paper is as follows. Section 2 reviews the Metis partitioning package and presents our modifications for circuit netlists. Section 3 presents our Metis-based ge- netic algorithm. Section 4 presents experimental results for $23 \mathrm{ACM} /$ SIGDA benchmarks, and Section 5 concludes with directions for future work.

\section{GRAPH PARTITIONING USING METIS}

The Metis package of Karypis and Kumar has multiple algorithm options for coarsening, for the initial partitioning step, and for refinement. For example, one can choose among eight different matching-based clustering schemes including random, heavy-edge, light-edge, and heavy-clique matching. The methodology we use follows the general recommendations of [13], even though their algorithm choices are based on extensive empirical studies of finite-element graphs and not circuit netlists. Before multilevel partitioning is performed, the adjacency lists for each module are randomly permuted. The following discussion applies our previous notation to weighted graphs; a weighted graph is simply a hypergraph $H_{i}$ and $|e|=2$ for each $e \in E_{i}$ with a nonnegative weight function $w$ on the edges.

To cluster, Karypis and Kumar suggest Heavy-Edge Matching (HEM), which is a variant of the random matching algorithm of [5]. A matching $M$ of $H_{i}$ is a subset of $E_{i}$ such that no module is incident to more than one edge in $M$. Each edge in the matching will be contracted to form a cluster, and the contracted edges should have the highest possible weights since they will not be cut in the graph $H_{i+1}$. HEM visits the modules in random order; if a module $u$ is unmatched, the edge $(u, v)$ is added to $M$ where $w(u, v)$ is maximum over all unmatched modules $v$; if $u$ has no unmatched neighbors, it remains unmatched. This greedy algorithm by no means guarantees a maximum sum of edge weights in $M$, but it runs in $O\left(\left|E_{i}\right|\right)$ time. Following [15], our methodology iteratively coarsens until $\left|V_{m}\right| \leq 100$.

An initial bipartitioning for $H_{m}$ is formed by the Greedy Graph Growing Partitioning (GGGP) algorithm. Initially, one "fixed" module $v$ is in its own cluster $X_{m}$ and the rest of the modules are in $Y_{m}$. Modules with highest gains are greedily moved from $Y_{m}$ to $X_{m}$ until $P_{m}=\left\{X_{m}, Y_{m}\right\}$ satisfies the cluster size constraints. Since the solution is extremely sensitive to the initial choice of $v$, the algorithm is run four times with different initial modules, and the best solution observed is retained for the next step. Despite its simplicity, the GGGP heuristic proved at least as effective as other heuristics for partitioning finite element graphs [13].

The refinement steps use the Boundary Kernighan-Lin Greedy Refinement (BGKLR) scheme. Despite its name, the heuristic actually uses the FM single-module neighborhood structure. Kumar and Karypis label the KL algorithm "greedy" when only a single pass is performed, and propose a hybrid algorithm which performs "complete" KL when the graph is small (i.e., less than 2000 modules) and greedy KL for larger graphs. They show that greedy KL is only slightly inferior to complete KL, but saves substantial CPU time. A "boundary" scheme is also used for updating gains: initially, only modules that are incident to cut edges (i.e., boundary modules) are stored in the FM bucket data structure and are eligible to be moved; when a module that is not in the data structure becomes incident to a moved module, it is inserted into the bucket data structure only if it has 
positive gain. The cost of performing the boundary version of KL is small, since only the boundary modules are considered. The overall Metis methodology is presented in Figure 2.

\begin{tabular}{|l|}
\hline The Metis Algorithm \\
\hline Input: Graph $H_{0}\left(V_{0}, E_{0}\right)$ \\
Output: Bipartitioning $P_{0}=\left\{X_{0}, Y_{0}\right\}$ \\
\hline 1. i $=0 ;$ randomly permute the adjacency lists of $H_{0}$. \\
2. while $\left|V_{i}\right|<100$ do \\
3. Use HEM to find a matching $M$ of $H_{i}$ \\
4. Contract each edge in $M$ to form a clustering. \\
! Construct the coarser graph $H_{i+1}\left(V_{i+1}, E_{i+1}\right)$. \\
Set $i$ to $i+1$. \\
5. Let $m=i$. Apply GGGP to $H_{m}$ to derive $P_{m}$. \\
Refine $P_{m}$ using BGKLR. \\
6. for $\mathrm{i}=\mathrm{m}-1$ downto 0 do \\
7. Project solution $P_{i+1}$ to the new solution $P_{i}$. \\
8. return $P_{0}$. \\
\hline
\end{tabular}

Figure 2. The Metis Algorithm

To run Metis on circuit netlists, we use an efficient hypergraph to graph converter that constructs sparse graphs. The traditional clique net model (which adds an edge to the graph for every pair of modules in a given net) is not a good choice since large nets will destroy sparsity. Since we observed that keeping large nets generally increases the cut size regardless of the net model, we removed all nets with more than $T$ modules (we use $T=50$ ). For each net $e$, our converter picks $F \cdot|e|$ random pairs of modules in $\epsilon$ and adds an edge with cost one into the graph for each pair. Here, $F$ is a constant; our experiments show that the value of $F$ is not too significant as long as it is large enough (we use $F=5$ ). Table 1 shows how the Metis cuts vary with various values of $F$ and $T$. Our converter retains the sparsity of the circuit, introduces randomness to allow multiple Metis runs, and is fairly efficient.

\section{A GENETIC VERSION OF METIS}

Our experiments in Section 4 show that over the course of 100 independent runs Metis generates at least one very good solution, but that its performance is not particularly stable, generating average cuts much higher than minimum cuts. To try to stabilize solution quality and generate superior solutions, we have integrated Metis into a genetic framework.

An indicator vector $\vec{p}=\left\{p_{1}, p_{2}, \ldots, p_{n}\right\}$ for a bipartitioning $P=\{X, Y\}$ has entry $p_{i}=0$ if $v_{i} \in X$ and entry $p_{i}=1$ if $v_{i} \in Y$, for all $i=1,2, \ldots, n$. The distance between two bipartitionings $P$ and $Q$ with corresponding indicator vectors $\vec{p}$ and $\vec{q}$ is given by $\sum_{i=1}^{n}\left|p_{i}-q_{i}\right|$, i.e., by the number of module moves needed to derive solution $Q$ from the initial solution $P$. Boese et al. [4] showed that the set of local minima generated by multiple FM runs exhibit a "big valley" structure: solutions with smallest distance to the lowest-cost local minima also have low cost, and the best local minima are "central" with respect to the other local minima. Thus, we seek to combine several local minimum solutions generated by Metis into a more "central" solution.
Given a set $S$ of $s$ solutions, the $s$-digit binary code $C(i)$ for module $v_{i}$ is generated by concatenating the $i^{\text {th }}$ entries of the indicator vectors for the $s$ solutions. We construct a clustering by assigning modules $v_{i}$ and $v_{j}$ to the same cluster if $C(i)$ and $C(j)$ are the same code. Our strategy integrates this code-generated clustering into Metis, in that we use HEM clustering and force every clustering generated during coarsening to be a refinement of the codebased clustering. ${ }^{2}$ Our Genetic Metis (GMetis) algorithm is shown in Figure 3.

\begin{tabular}{|l|}
\hline The Genetic Metis (GMetis) Algorithm \\
\hline Input: $\quad$ Hypergraph $H(V, E)$ with $n$ modules \\
Output: $\quad$ Bipartitioning $P=\{X, Y\}$
\end{tabular}

Figure 3. The Genetic Metis Algorithm

Step 1 initially sets all codes to $00 \ldots 0$ which causes GMetis to behave just like Metis until $s$ solutions are generated. Steps 2 and 3 are loops which cause numgen generations of $s$ solutions to be computed. Next, Step 4 converts the circuit hypergraph into a graph, but this step is performed only once out of every 10 times Metis is called. We perform the conversion with this frequency to reduce runtimes while still allowing a variety of different graph representations; the constant 10 is fairly arbitrary. In Step 5, Metis is called using our version of HEM described above. Step 6 maintains the set of solutions $S$; our replacement scheme replaces solution $Q \in S$ with solution $P$ if $P$ has smaller cut size than $Q$; other replacement schemes may work just as well and need to be investigated. Step $7 \mathrm{com}-$ putes the binary code for each module based on the current solution set, but only after the first generation has completed and five solutions with the previous code-based clustering have been generated. As in Step 4, the constant 5 is fairly arbitrary. Finally, the solution with lowest cut is returned in Step 8.

\section{EXPERIMENTAL RESULTS}

All of our experiments use a subset of the benchmarks from the ACM/SIGDA suite given in Table 2; hypergraph formats of these circuits are available on the world wide web

\footnotetext{
${ }^{2}$ A clustering $P^{k}$ is a refinement of $Q^{l}(k \geq l)$ if some division of clusters in $Q^{l}$ will yield $P^{k}$.
} 


\begin{tabular}{|c|c|c|c|c|c|c|c|c|c|c|}
\hline$T / F$ & 1 & 2 & 3 & 4 & 5 & 6 & 8 & 10 & 12 & 15 \\
\hline 10 & $289(238)$ & $241(184)$ & $239(185)$ & $238(180)$ & $225(176)$ & $228(184)$ & $230(174)$ & $220(169)$ & $225(178)$ & $227(169)$ \\
15 & $276(231)$ & $224(188)$ & $239(185)$ & $228(184)$ & $222(178)$ & $228(175)$ & $228(165)$ & $215(176)$ & $241(181)$ & $228(174)$ \\
20 & $320(261)$ & $252(202)$ & $259(180)$ & $258(189)$ & $252(173)$ & $253(187)$ & $261(165)$ & $269(190)$ & $265(176)$ & $253(178)$ \\
25 & $321(243)$ & $251(189)$ & $250(174)$ & $254(170)$ & $243(169)$ & $238(162)$ & $255(173)$ & $232(162)$ & $245(176)$ & $266(174)$ \\
35 & $309(243)$ & $248(172)$ & $239(170)$ & $227(168)$ & $249(173)$ & $245(171)$ & $240(166)$ & $247(164)$ & $254(176)$ & $239(169)$ \\
50 & $316(233)$ & $258(190)$ & $250(177)$ & $251(173)$ & $245(169)$ & $240(167)$ & $255(159)$ & $255(178)$ & $232(162)$ & $240(175)$ \\
100 & $310(231)$ & $274(184)$ & $256(173)$ & $260(172)$ & $256(173)$ & $254(175)$ & $248(166)$ & $237(170)$ & $245(180)$ & $252(176)$ \\
200 & $325(252)$ & $265(182)$ & $266(170)$ & $257(174)$ & $288(184)$ & $258(182)$ & $261(187)$ & $260(192)$ & $271(181)$ & $266(186)$ \\
500 & $471(366)$ & $427(333)$ & $418(318)$ & $412(327)$ & $414(294)$ & $429(295)$ & $399(311)$ & $408(321)$ & $414(296)$ & $411(270)$ \\
\hline
\end{tabular}

Table 1. Average(minimum) cuts for the avqlarge test case for 50 runs of Metis shown for various values of $T$ (rows) and $F$ (columns).

at http://ballade.cs.ucla.edu/ ${ }^{2}$ cheese. Our experiments assume unit module areas, and our code was written in $\mathrm{C}++$ and was compiled with $\mathrm{g}++v 2.4$ on a Unix platform. Our experiments were run on an $85 \mathrm{Mhz}$ Sun Sparc 5 and all runtimes reported are for this machine (in seconds) unless otherwise specified. We performed the following studies:

- We compare Metis against standard and two-phase FM, to show the effectiveness of the multilevel approach.

- We show that the GMetis algorithm is more effective than running Metis multiple times.

- Finally, we show that GMetis is competitive with previous approaches while using a fraction of the runtime.

\begin{tabular}{|c|c|c|c|}
\hline Test Case & \# Modules & \# Nets & \# Pins \\
\hline balu & 801 & 735 & 2697 \\
bm1 & 882 & 903 & 2910 \\
primary1 & 833 & 902 & 2908 \\
test04 & 1515 & 1658 & 5975 \\
test03 & 1607 & 1618 & 5807 \\
test02 & 1663 & 1720 & 6134 \\
test06 & 1752 & 1541 & 6638 \\
struct & 1952 & 1920 & 5471 \\
test05 & 2595 & 2750 & 10076 \\
19ks & 2844 & 3282 & 10547 \\
primary2 & 3014 & 3029 & 11219 \\
s9234 & 5866 & 5844 & 14065 \\
biomed & 6514 & 5742 & 21040 \\
s13207 & 8772 & 8651 & 20606 \\
s15850 & 10470 & 10383 & 24712 \\
industry2 & 12637 & 13419 & 48404 \\
industry3 & 15406 & 21923 & 65792 \\
s35932 & 18148 & 17828 & 48145 \\
s38584 & 20995 & 20717 & 55203 \\
avqsmall & 21918 & 22124 & 76231 \\
s38417 & 23849 & 23843 & 57613 \\
avqlarge & 25178 & 25384 & 82751 \\
golem3 & 103048 & 144949 & 338419 \\
\hline
\end{tabular}

Table 2. Benchmark circuit characteristics.

\subsection{Metis vs. FM and Two-phase FM}

Our first set of experiments compares Metis against both FM and two-phase FM. We ran Metis 100 times with balance parameter $r=0$ (exact bisection) and recorded the minimum cut observed in the second column of Table 3 . Since there are many implementations of FM (some of which are better than others), we compare to the best FM results found in the literature.

\begin{tabular}{|c||c|c|c|c||c|c|}
\hline \multicolumn{1}{|c||}{\multirow{2}{*}{ Test }} \\
Case & \multicolumn{3}{c||}{ Minimum cut (100 runs) } & \multicolumn{2}{c|}{ CPU (s) } \\
\cline { 2 - 7 } & Metis & FM & FM & 2-FM & Metis & FM \\
\cline { 2 - 7 } & & {$[6]$} & {$[9]$} & $[1] 9]$ & & {$[6]$} \\
\hline \hline balu & 34 & 32 & & & 23 & 21 \\
bm1 & 53 & 55 & & 52 & 22 & 24 \\
primary1 & 55 & 57 & 56 & 53 & 22 & 24 \\
test04 & 53 & 86 & & 56 & 37 & 41 \\
test03 & 61 & 72 & & 60 & 39 & 56 \\
test02 & 99 & 115 & & 97 & 43 & 46 \\
test06 & 94 & 71 & & 68 & 53 & 50 \\
struct & 36 & 45 & 36 & 43 & 41 & 46 \\
test05 & 107 & 97 & & 93 & 69 & 81 \\
19ks & 116 & 142 & & 121 & 59 & 115 \\
primary2 & 158 & 236 & 171 & 182 & 90 & 128 \\
s9234 & 49 & 53 & & & 72 & 222 \\
biomed & 83 & 83 & 83 & 124 & 134 & 296 \\
s13207 & 84 & 92 & & & 111 & 339 \\
s15850 & 62 & 112 & & & 123 & 339 \\
industry2 & 218 & 428 & 275 & 438 & 349 & 727 \\
industry3 & 292 & & 312 & 328 & 399 & \\
avqsmall & 175 & & 373 & 399 & 293 & \\
avqlarge & 171 & & 406 & 518 & 355 & \\
\hline
\end{tabular}

Table 3. Comparison of Metis with FM.

Dutt and Deng [6] have implemented very efficient FM code; their exact bisection results for the best of $100 \mathrm{FM}$ runs are given in the third column of Table 3 and the corresponding Sparc 5 run times are given in the last column. Hagen et al. [9] have run FM with an efficient LIFO tie breaking strategy and a new lookahead function that outperforms [17]; their bisection results are reported in the fourth column. Finally, we compare to various two-phase FM strategies. In the fifth column, we give the best twophase FM results observed for various clustering algorithms as reported in [1] and [9].

Metis does not appear to be faster than FM for circuits with less than two thousand modules, but for larger circuits with five to twelve thousand modules, Metis is 2-3 times faster. In terms of cut sizes, again Metis is indistinguishable from FM for the smaller benchmarks, but Metis cuts are significantly lower for the larger benchmarks. We conclude that multilevel approaches are unnecessary for small circuits, but greatly enhance solution quality for larger circuits. For these circuits, more than two levels of clustering are required for such an iterative approach to be effective. 


\subsection{Genetic Metis vs. Metis}

The next set of experiments compares Metis with the GMetis. We ran GMetis for 10 generations while maintaining $s=10$ solutions so that both Metis and GMetis considered 100 total solutions. The minimum and average cuts observed, as well as total CPU time, are reported for both algorithms in Table 4.

\begin{tabular}{|c||c|c|c||c|c|c|}
\hline \multicolumn{1}{|c||}{ Test } & \multicolumn{3}{c||}{ Metis } & \multicolumn{3}{c|}{ GMetis } \\
\cline { 2 - 7 } Case & min & avg & CPU & min & avg & CPU \\
\hline \hline balu & 34 & 47 & 26 & 32 & 38 & 24 \\
bm1 & 53 & 65 & 23 & 54 & 59 & 22 \\
primary1 & 55 & 66 & 23 & 55 & 59 & 21 \\
test04 & 53 & 68 & 37 & 52 & 58 & 37 \\
test03 & 61 & 76 & 42 & 65 & 74 & 39 \\
test02 & 99 & 113 & 44 & 96 & 101 & 42 \\
test06 & 94 & 117 & 59 & 97 & 121 & 55 \\
struct & 36 & 52 & 41 & 34 & 40 & 39 \\
test05 & 107 & 125 & 70 & 109 & 117 & 69 \\
19ks & 116 & 132 & 59 & 112 & 116 & 59 \\
primary2 & 158 & 195 & 95 & 165 & 174 & 91 \\
s9234 & 49 & 66 & 71 & 45 & 52 & 68 \\
biomed & 83 & 149 & 145 & 83 & 134 & 143 \\
s13207 & 84 & 90 & 106 & 78 & 89 & 112 \\
s15850 & 62 & 84 & 126 & 59 & 74 & 125 \\
industry2 & 218 & 280 & 336 & 204 & 230 & 339 \\
industry3 & 292 & 408 & 384 & 291 & 313 & 423 \\
s35932 & 55 & 71 & 257 & 56 & 62 & 265 \\
s38584 & 55 & 101 & 310 & 53 & 67 & 368 \\
avqsmall & 175 & 241 & 289 & 148 & 174 & 322 \\
s38417 & 73 & 110 & 294 & 74 & 104 & 301 \\
avqlarge & 171 & 248 & 318 & 144 & 181 & 355 \\
golem3 & 2196 & 2520 & 1592 & 2196 & 2648 & 1928 \\
\hline
\end{tabular}

Table 4. Comparison of Metis with Genetic Metis. The minimum cut, average cut and CPU time for 100 runs of each algorithm are given.

On average, GMetis yields minimum cuts that are $2.7 \%$ lower than Metis, and significantly lower average cuts (except for golem 3). For the larger benchmarks (seven to twenty-six thousand modules) GMetis cuts are 5.7\% lower, with significant improvements for industry2, avqsmall and avqlarge. We believe that GMetis can have the greatest impact for larger circuits. Note that golem 3 is the one benchmark in which Metis outperforms GMetis in terms of the average case - the quality of GMetis solutions gradually became worse in subsequent generations instead of converging to single good solutions.

\subsection{Genetic Metis vs. Other Approaches}

Finally, we compare GMetis to other recent partitioning works in the literature, namely PROP [6], Paraboli [20], and GFM [19], the results of which are quoted from the original sources and presented in Table 5. All these works use $r=0.1$, i.e., each cluster contains between $45 \%$ and $55 \%$ of the total number of modules. The CPU times in seconds for PROP, Paraboli, and GFM are respectively reported for a Sun Sparc 5, a DEC 3000 Model 500 AXP, and a Sun Sparc 10. We modified GMetis to handle varying size constraints by allowing the BGKLR algorithm to move modules while satisfying the cluster size constraints. We found that with this implementation, GMetis with $r=0.1$ was sometimes outperformed by GMetis with $r=0$ (exact bisection). Hence, in Table 5, we present results for GMetis with $r=0.1$ and $r=0$ (given in parentheses). ${ }^{3}$ Since for $r=0.1$, GMetis runtimes sometimes increase by $20-50 \%$, we report runtimes for $r=0$ in the last column. These experiments used the somewhat arbitrary parameter values of $s=\log _{2} n(|V|=n)$ solutions and 12 generations.

Observe that GMetis cuts are competitive with the other methods, especially for the larger benchmarks s15850, industry2, and avqsmall. However, the big win for GMetis is the short runtimes: generating a single solution for avqlarge and golem 3 respectively takes $417 /\left(12 \log _{2} 25178\right)=2.5$ and $450 /\left(2 \log _{2} 103048\right)=15$ seconds on average. For golem 3 , we only ran 2 generations since the results do not improve with subsequent generations; the solution with cost 2144 was achieved after only 210 seconds of CPU time.

\section{CONCLUSIONS}

This work integrates the Metis multilevel partitioning algorithm of [15] into a genetic algorithm. We showed (i) Metis outperforms previous FM-based approaches, (ii) GMetis improves upon Metis alone for large benchmarks, and (iii) GMetis is competitive with previous approaches while using less CPU time. There are many improvements which we are pursuing:

- Find sparser and more reliable hypergraph conversion algorithms.

- Try alternative genetic replacement schemes, instead of simply inserting the current solution into $S$ if it is a better solution.

- Tweak parameters such as $F, T, s$, and the number of generations in order to generate more stable solutions in fewer iterations.

- Experiment with various schemes to control cluster sizes in the bipartitioning solution. That GMetis frequently finds better $50-50$ solutions versus $45-55$ solutions is not acceptable.

- Finally, we are integrating our own separate multilevel circuit partitioning code into a new cell placement algorithm.

\section{REFERENCES}

[1] C. J. Alpert and A. B. Kahng, "A General Framework for Vertex Orderings, with Applications to Netlist Clustering", to appear in IEEE Trans. on VLSI.

[2] C. J. Alpert and A. B. Kahng, "Recent Directions in Netlist Partitioning: A Survey", Integration, the VLSI Journal, 19(1-2), pp. 1-81, 1995.

[3] C. J. Alpert and S.-Z. Yao, "Spectral Partitioning: The More Eigenvectors, the Better," Proc. ACM/IEEE Design Automation Conf., 1995, pp. 195-200.

[4] K. D. Boese, A. B. Kahng, and S. Muddu, "A New Adaptive Multi-Start Technique for Combinatorial Global Optimizations", Operations Research Letters, 16(20), pp. 101-13, 1994.

${ }^{3}$ We attribute this undesirable behavior to improper modification of the Metis code. We believe that a better implementation should yield $r=0.1$ results at least as good as those for $r=0$ without increasing runtimes. 


\begin{tabular}{|c|c|c|c|c|c|c|c|c|}
\hline \multirow{2}{*}{$\begin{array}{l}\text { Test } \\
\text { Case }\end{array}$} & \multicolumn{4}{|c|}{ cuts } & \multicolumn{4}{|c|}{$\mathrm{CPU}$} \\
\hline & PROP & Paraboli & GFM & GMetis(baI) & PROP & Paraboli & GFM & GMetis \\
\hline balu & 27 & 41 & 27 & $27(32)$ & 16 & 16 & 24 & $\overline{14}$ \\
\hline bm1 & 50 & & & $48(53)$ & 20 & & & 12 \\
\hline primary 1 & 47 & 53 & 47 & $47(54)$ & 19 & 18 & 16 & 12 \\
\hline test 04 & 52 & & & $49(52)$ & 49 & & & 21 \\
\hline test 03 & 59 & & & $62(66)$ & 51 & & & 23 \\
\hline test 02 & 90 & & & $95(96)$ & 64 & & & 26 \\
\hline test 06 & 76 & & & $94(93)$ & 75 & & & 32 \\
\hline struct & 33 & 40 & 41 & $33(34)$ & 42 & 35 & 80 & 27 \\
\hline test 05 & 79 & & & $104(109)$ & 97 & & & 46 \\
\hline $19 \mathrm{ks}$ & 105 & & & $106(110)$ & 87 & & & 39 \\
\hline primary 2 & 143 & 146 & 139 & $142(158)$ & 139 & 137 & 224 & 53 \\
\hline s9234 & 41 & 74 & 41 & $43(45)$ & 139 & 490 & 672 & 58 \\
\hline biomed & 83 & 135 & 84 & $102(83)$ & 250 & 711 & 1440 & 95 \\
\hline s13207 & 75 & 91 & 66 & $74(70)$ & 177 & 2060 & 1920 & 102 \\
\hline s15850 & 65 & 91 & 63 & $53(60)$ & 291 & 1731 & 2560 & 114 \\
\hline industry 2 & 220 & 193 & 211 & $177(204)$ & 867 & 1367 & 4320 & 245 \\
\hline industry 3 & & 267 & 241 & $243(286)$ & & 761 & 4000 & 299 \\
\hline s35932 & & 62 & 41 & $57(55)$ & & 2627 & 10160 & 266 \\
\hline s38584 & & 55 & 47 & $53(53)$ & & 6518 & 9680 & 397 \\
\hline avqsmall & & 224 & & $144(145)$ & & 4099 & & 328 \\
\hline s 38417 & & 49 & 81 & $69(77)$ & & 2042 & 11280 & 281 \\
\hline avqlarge & & 139 & & $145(144)$ & & 4135 & & 417 \\
\hline golem 3 & & 1629 & & $2111(2144)$ & & 10823 & & 450 \\
\hline
\end{tabular}

Table 5. Comparison of GMetis with PROP, Paraboli, and GFM for min-cut bipartitioning allowing $10 \%$ deviation from bisection. Exact bisection results for GMetis are given in parentheses in the fifth column.

[5] T. Bui, C. Heigham, C. Jones, and T. Leighton, "Improving the Performance of the Kernighan-Lin and Simulated Annealing Graph Bisection Algorithms", Proc. ACM/IEEE Design Automation Conf., pp. 775778,1989

[6] S. Dutt and W. Deng, "A Probability-Based Approach to VLSI Circuit Partitioning", to appear in Proc. ACM/IEEE Design Automation Conf., 1996.

[7] S. Dutt and W. Deng, "VLSI Circuit Partitioning by Cluster-Removal Using Iterative Improvement Techniques", Technical Report, Department of Electrical Engineering, University of Minnesota, Nov. 1995.

[8] C. M. Fiduccia and R. M. Mattheyses, "A Linear Time Heuristic for Improving Network Partitions", Proc. ACM/IEEE Design Automation Conf., pp. 175-181, 1982.

[9] L. W. Hagen, D. J.-H. Huang, and A. B. Kahng, "On Implementation Choices for Iterative Improvement Partitioning Algorithms", to appear in IEEE Trans. Computer-Aided Design (see also Proc. European Design Automation Conf., Sept. 1995, pp. 144149).

[10] S. Hauck and G. Borriello, "An Evaluation of Bipartitioning Techniques", Proc. Chapel Hill Conf. on Adv. Research in VLSI, 1995.

[11] B. Hendrickson and R. Leland, "A Multilevel Algorithm for Partitioning Graphs", Technical Report SAND93-1301, Sandia National Laboratories, 1993.

[12] B. Hendrickson and R. Leland, "The Chaco User's Guide", Technical Report SAND93-2339, Sandia National Laboratories, 1993.

[13] G. Karypis and V. Kumar, "A Fast and High Quality Multilevel Scheme for Partitioning Irregular Graphs",
Technical Report \#95-035, Department of Computer Science, University of Minnesota, 1995.

[14] G. Karypis and V. Kumar, "Multilevel k-Way Partitioning Scheme for Irregular Graphs", Technical Report \#95-035, Department of Computer Science, University of Minnesota, 1995.

[15] G. Karypis and V. Kumar, "Unstructured Graph Partitioning and Sparse Matrix Ordering", Technical Report, Department of Computer Science, University of Minnesota, 1995 (see http://www.cs.umn.edu/ ^kumar for postscript and code).

[16] B. W. Kernighan and S. Lin, "An Efficient Heuristic Procedure for Partitioning Graphs", Bell Systems Tech. J., 49(2), pp. 291-307, 1970.

[17] B. Krishnamurthy, "An Improved Min-Cut Algorithm for Partitioning VLSI Networks", IEEE Trans. Computers, 33(5), pp. 438-446, 1984.

[18] J. Li, J. Lillis, and C.-K. Cheng, "Linear Decomposition Algorithm for VLSI Design Applications", Proc. IEEE Intl. Conf. Computer-Aided Design, pp. 223-228, 1995.

[19] L.-T. Liu, M.-T. Kuo, S.-C. Huang, and C.-K. Cheng, "A Gradient Method on the Initial Partition of Fiduccia-Mattheyses Algorithm", Proc. IEEE Intl. Conf. Computer-Aided Design, pp. 229-234, 1995.

[20] B. M. Riess, K. Doll, and F. M. Johannes, "Partitioning Very Large Circuits Using Analytical Placement Techniques", Proc. ACM/IEEE Design Automation Conf., pp. 646-651, 1994.

[21] Y. Saab, "A Fast and Robust Network Bisection Algorithm", IEEE Trans. Computers, 44(7), pp. 903-913, 1995.

[22] L. A. Sanchis, "Multiple-Way Network Partitioning", IEEE Trans. Computers, 38(1), pp. 62-81, 1989. 